\title{
Operating parameters for a CAM system
}

When you use a CAM system, you need to set up some additional parameters in the initial start-up procedure, over and above those required by a CAD system (discussed in Learning Assignment 9 ). These parameters relate to the ultimate generation of a CNC program to machine the drawn component and may include the following:

- the data directory - the directory for storing drawing profile and CNC data files; this allows data for different types of work, or for work for different customers, to be separated and stored in their own directories on disc

- the machine directory - the directory for storing post-processors, backplot files and tooling information; this allows data for different CNC machines to be stored in their own directory, such as Fanuc OM for milling, or Fanuc OT for turning

- the tool library, either milling tools or turning tools

- the canned cycle call - an option to use the canned cycle calls for the CNC machine tool set in the machine directory.

\section{Task 10.1 Starting on a CAM system}

Select and step through the parameter settings in your CAD/CAM system and make a list of the settings suitable for the component shown in Figure 8.1 (page 36). It may be that your system will answer some or all of these questions because parameters may already be 'set up' as you progress through the system. Obviously, settings vary from one CAM system to another. Here, however, is a list of settings for Precision CAD/CAM software:

drawing size

units

number of decimal places

A2

scale

$\mathrm{mm}$

$1: 1$

co-ordinate origin

$\mathrm{X} 0.000 \quad \mathrm{Y} 0.000$

number of decimal places

machine directory

3

data directory

tool library

canned cycle calls

Fanuc OM

Runcorn Engineering milling tools

importing drawing datas from a CAD system DXF 